\title{
Refinements to the Variety Metric for Idea Evaluation
}

\begin{abstract}
Systematically developing innovative products demands effective idea generation methods. The effectiveness can be verified based on a set of metrics, of which the variety metric is one. It is demonstrated that this metric exhibits several shortcomings, such as the lack of level-based measurements, and arbitrarily defined level weights. The currently applied variety metrics, furthermore, do not measure the degree of uniformness of the distribution of ideas over nodes on an abstraction level. A level-based, correctly normalized variety metric which accounts for the degree of uniformness of the distribution of concepts over nodes, is proposed, and is shown to resolve the above issues.
\end{abstract}

Keywords: engineering design, conceptual design, creativity, evaluation, variety

According to Milton (2003), top innovating companies can generate over $75 \%$ of their revenue from products and services that were not in existence five years ago, indicating that, for an enterprise, one of the ways to maintain its competitive advantage is to develop innovative products. It is furthermore commonly believed that design decisions determine up to $70 \%$ of total product costs (Dowlatshahi, 1992). Although the exact figure is disputed (Ulrich \& Pearson, 1993; Barton et al., 2001), it is safe to say that the cost correlates strongly to the early phases of the engineering design process, such as the 
problem definition, the information gathering and the idea generation phase. For the latter phase, a plethora of idea generation methods exist (Silverstein et al., 2008). The effectiveness of these methods is mostly claimed based on anecdotal evidence, and only recently formal experiments have been set up to prove and compare their effectiveness (Linsey, 2007; Weaver et al., 2009), and to delineate their proper usage conditions (Linsey, 2007).

In process-based studies, the effectiveness of these methods is analyzed in controlled experiments through the observation of specific cognitive processes. These experiments are time-consuming, inherently subjective (Shah et al., 2000), and their external validity is highly uncertain when extrapolating the results to more complex engineering problems (Shah et al., 2000). For these reasons, outcome-based studies, in which the effectiveness of idea generation methods is solely based on the generated designs, are predominant (Shah et al. 2000). In outcome-based studies, the different aspects of the generated designs, and the idea space formed by these designs, are quantified by a number of metrics. This research proposes refinements to one of the existing metrics, the variety metric, in order to allow for more detailed analyses of the variety at each abstraction level, and to account for the degree of uniformness of the distribution of ideas over the nodes.

The next section offers an overview of the related research on the different idea evaluation metrics. Section 2 details the existing variety metrics. Section 3 identifies the problems with the current variety metrics by means of examples, and proposes refinements to the variety metric. Section 4 formulates conclusions, and the final section outlines possible future research trajectories. 


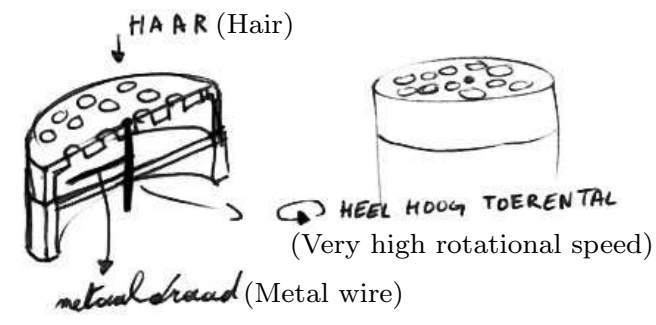

(a) Shaver concept in which hair is cut by a metal wire at a very high rotational speed.

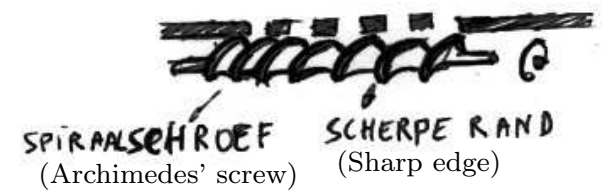

(b) Shaver concept in which hair is cut by an Archimedes' screw with a sharp edge.

Figure 1: Two sketches of concepts generated for a new electric shaver. Translations of the handwritten Dutch words are added in parentheses.

\section{Related Research}

\subsection{Definitions}

In the context of this research, a concept is defined as a set of ideas (each from a different level of abstraction), and their relationships, which are as a whole expected to perform one or more required functions as subject of an identified problem. A concept can be represented by sketches or wordings, as illustrated by Figure 1 depicting two sketches of concepts generated for a new electric shaver. Design sketches typically focus on the aspects necessary to convey a concept without providing an illustration of the complete product or appliance. Figure 1a, for instance, shows a sketch of a concept involving a metal wire that cuts hair as it rotates at a high speed, but does not depict non-essential elements such as a handle or an electric motor.

An idea is the representation of a concept at a certain level of abstraction. A common set of recognized abstraction levels, also often referred to in this research, are the physical principle, the working principle, the embodiment and the detail level (Shah et al. 2000; Linsey, 2007). Figure 1a and Figure 
$1 \mathrm{~b}$ show two concepts sharing the same idea of cutting to remove hair at the physical principle abstraction level. Other concepts can use other ideas to remove hair at the physical level, e.g. burning hair or dissolving hair chemically. At a working level, however, the two concepts depicted in Figure 1 apply different ideas, e.g. Figure 1a depicts a concept applying the idea of planar rotatory motion to cut, while Figure $1 \mathrm{~b}$ shows a helical rotating motion as a working principle level idea. At the embodiment level, an idea typically stands for one or more parts, e.g. using a rotating wire to cut, or using the Archimedes' screw to cut. The lowest, or detail, level can be thought of as a blueprint of the concept, e.g. exact description and dimensions of the screw used to cut.

The definitions of a concept and an idea are based on previous research by other authors. Srinivasan \& Chakrabarti (2010) propose similar definitions, although implemented on a different set of abstraction levels based on the SAPPhIRE model (see Section 2.2.5). According to Linsey (2007) an idea must provide one or more of the functions as defined by the functional basis proposed by Hirtz et al. (2001). Apart from this more strict definition of a function, these definitions are similar to the definitions applied in this research. The definitions of an idea and a solution method in Shah et al. (2003) are similar respectively to the definition of a concept and an idea in this research. The definitions are also in agreement with Jansson \& Smith (1991) explicitly including ideas as well as relationships in concept space.

\subsection{Existing metrics}

Figure 2 provides a high-level overview of the different metrics for measuring the creativeness of ideas and ideation methods based on Dean et al. 


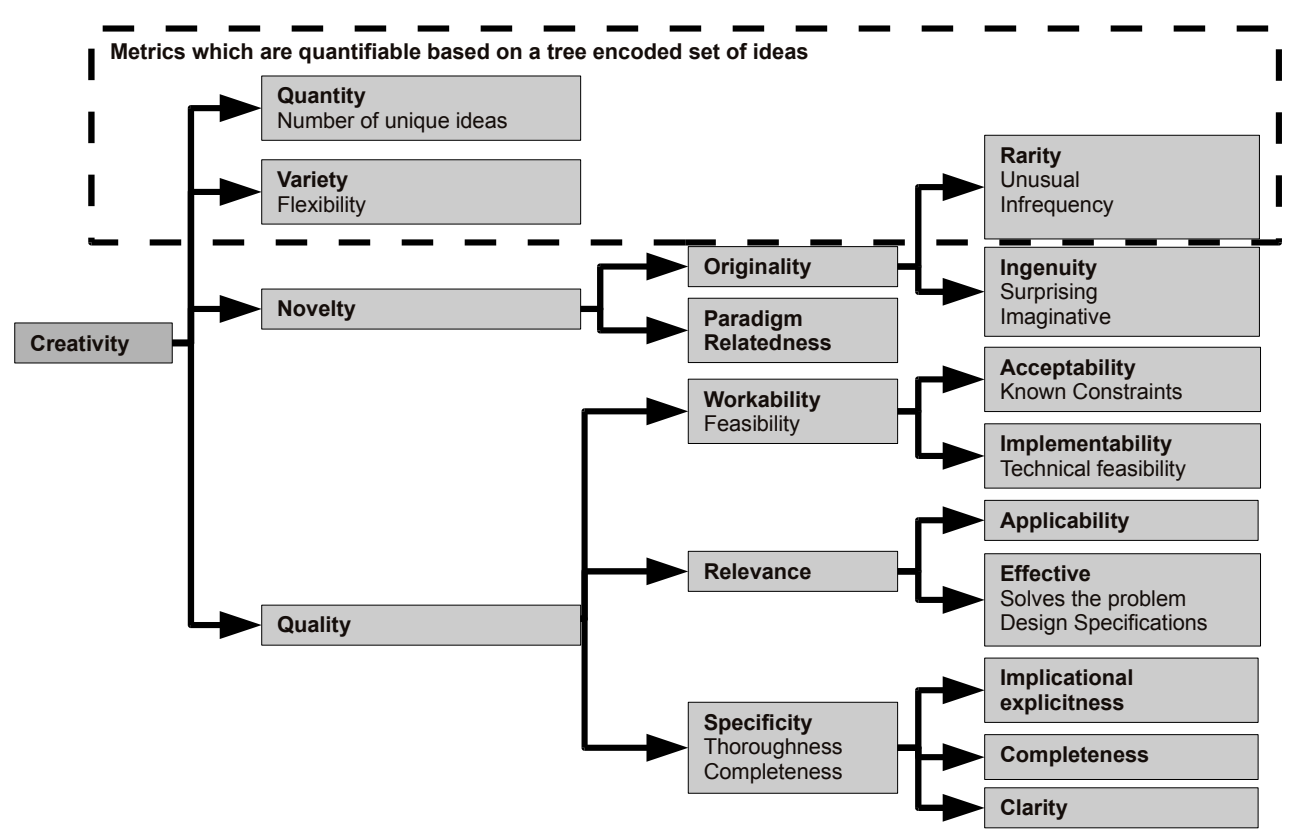

Figure 2: Overview of ideation effectiveness metrics.

(2006). However, in contrast to Dean et al., but in accordance to Shah et al. (2000), novelty is not part of quality. Figure 2 furthermore includes the variety proposed by Shah et al., which is also not covered by Dean et al. Figure 2 depicts multiple names for the metrics, since the terminology used for the different metrics is inconsistently applied among researchers. This overview is meant as a high-level guideline to map and compare metrics between different studies. The subsections below provide a more in-depth explanation of the respective metrics.

\subsubsection{Quantity metric}

Early work by MacCrimmon \& Wagner (1994) gives an overview of the applied quantitative and qualitative measures for idea generation in the context of group decision support systems studies, and states that the quantity or 
the number of generated unique concepts is a commonly agreed upon metric. Different protocols for determining the quantity of concepts were developed (Bouchard \& Hare, 1970; Dean et al., 2006). Later work by Linsey et al. (2005) in the domain of ideation method evaluation presents a formal procedure to quantify the number of unique concepts.

The quantity is a metric on a set of concepts. This metric is not correlated with the average quality of the generated concepts (Diehl \& Stroebe, 1987) unless the participants are forced to deeply explore subdomains in which case a positive correlation is recorded (Rietzschel, 2005). In unrestricted experiments, a higher number of concepts leads to an increase in the quality of individual concepts after selection, since every generated idea is believed to have an equal chance to be of high quality (Rietzschel, 2005). This is reflected in the brainstorming's mantra "Quantity breeds Quality" (Osborn, 1957). A number of studies have provided further proof of this positive correlation between the number of ideas on the one hand, and the total quality of all ideas, or retained average quantity of the ideas after selection on the other hand (Diehl \& Stroebe, 1987; MacCrimmon \& Wagner, 1994).

\subsubsection{Variety}

In the domain of design fixation, early work from Jansson \& Smith (1991) defines the flexibility for a participant as the number of different approaches to the design problem divided by the total number of designs for that participant. Although not explicitly defined, in this definition, a design and an approach can be interpreted as, respectively, a concept and an idea at a

high abstraction level. A low flexibility is interpreted as a narrow range of generated ideas, while a high flexibility is indicative of a broadly searched 
idea space.

Shah et al. (2000) propose variety as a more formal measure of the explored concept space during idea generation. This metric is applied to an encoded tree-like structure of the idea space, and gives higher weights to differentiation occurring in higher abstraction levels. In later research, Nelson et al. (2009) and Srinivasan \& Chakrabarti (2010) propose refinements to the variety metric, which are detailed further in Section 2.

\subsubsection{Novelty}

Dean et al. (2006) define novelty as a dimension of a more encompassing quality metric. Novelty is further subdivided in originality and paradigm relatedness. The former expresses the degree to which an idea is not only rare, but is also ingenious, imaginative, or surprising, while the latter expresses the degree to which an idea is radical or transformational.

Since rarity can be quantified, other research has used it as a measure in its own right (Connolly et al., 1990), as substitute for originality (Jansson $\&$ Smith, 1991), or as substitute for the novelty metric as a whole (Shah et al., 2000, Linsey, 2007). Peeters et al. (2010) propose to calculate the novelty for each abstraction level leading to a more fine-grained analysis of the effectiveness of the applied idea generation method.

The originality and paradigm relatedness should ideally be calculated based on a universe of all relevant ideas to compare against. This universe is fictitious, but Shah et al. (2000) propose two approaches to estimate the originality. The universe for comparison can be defined as all preconceived ideas assembled by experts before analyzing the results of the experiments. Another approach is to define the universe for comparison as the set of all 
ideas generated by the experiment participants themselves. Shah et al. (2003) demonstrate that the second approach allows to automatically calculate the originality after encoding the ideas in a tree-like structure. The second approach is however not without limitations when applied to systematic innovation tools aiming to generate only few high quality ideas, such as TRIZ Altshuller, 1999). In this case, the universe of comparison can not be built from the generated concepts, but should be built using the first approach, or by applying the second approach using another technique as baseline, such as brainstorming (Peeters, 2010).

\subsubsection{Quality}

Dean et al. (2006) propose the quality metric with subdimensions workability, relevance, specificity, and novelty. In some studies, quality is defined as the technical feasibility of an idea and how well it meets the design specifications (Shah et al. 2000). Due to the inherent subjectiveness, the latter is however also often disregarded in idea generation method research (Linsey, 2007). In these studies, quality is synonymous for technical feasibility or implementability, which can be coded through a more clearly defined scale (Linsey, 2007).

The quality of a set of concepts is sometimes calculated as the sum of the quality of each concept, often referred to as the total quality. Some studies recommend calculating the quality of a set of concepts as the average of the quality of all concepts, sometimes referred to as the average quality (Rietzschel, 2005), while other studies recommend defining quality as the highest quality, or average of the quality of all concepts above a certain quality threshold (Diehl \& Stroebe, 1987; Girotra et al., 2010). 
As detailed above, other research (Connolly et al., 1990, Jansson \& Smith, 1991; Shah et al., 2000; Linsey, 2007; Srinivasan \& Chakrabarti, 2010) has treated the novelty metric as a separate dimension. This categorization, also depicted in Figure 2, seems to be preferred in the domain of ideation method evaluation. The paragraphs below detail the other subdimensions of the quality metric.

Workability. Figure 2 adheres to the subdivision of workability in acceptability and implementability as proposed by Dean et al. (2006). The acceptability metric is the degree to which a concept is socially, legally or politically acceptable, while the implementability metric expresses how well an idea can be applied from a technical perspective.

Relevance. Relevance is further divided in applicability and effectiveness as proposed by Dean et al. (2006). Applicability is the degree to which the concept applies to the problem at hand, while the effectiveness expresses the degree to which the concept is expected to solve this problem. A more detailed overview and explanation of these metrics can be found in Dean et al. (2006).

Specificity. MacCrimmon \& Wagner (1994) measure the thoroughness, also called the specificity (Dean et al., 2006), or the completeness of a concept, a criterion originally adopted from the U.S. Patent Register regulations. This metric expresses the extent to which a concept is clearly, concisely, and exactly detailed, and can be measured by experts based on a predefined scale (Dean et al., 2006). Based on the concept of deferring concept evaluation (Osborn, 1957), it can be argued that specificity is less suitable for measur- 


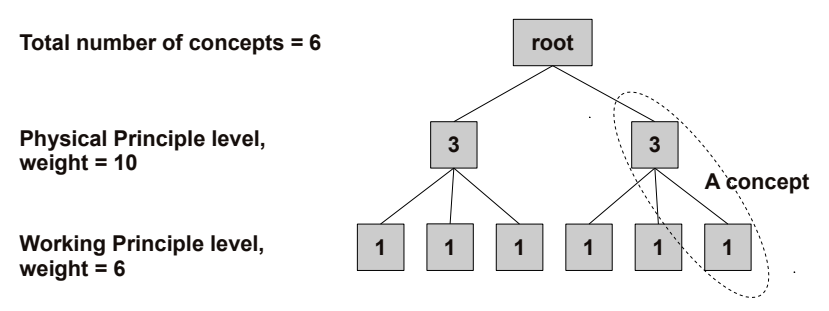

Figure 3: Example of the genealogy tree of a concept set.

ing the effectiveness of an ideation method. This might explain the fact that specificity is a less widely used metric.

\section{Existing Variety Metrics}

\subsection{Variety proposed by Shah}

Shah et al. (2000) propose the variety metric as a formal measure of the explored concept space. The generated concepts are encoded in a tree structure. Figure 3 illustrates such an idea genealogy tree with 6 concepts, each detailed by one idea at the working principle level, and grouped in two separate ideas at the physical principle level. Each node in the tree represents an idea, and the dotted ellipse in this figure exemplifies one of the six concepts as a combination of an idea at the physical principle level with an idea at the working principle level. A genealogy tree is constructed for each system function to improve. The variety is calculated from

$$
V=10 \sum_{j=1}^{m} f_{j} \sum_{k=1}^{4} \frac{S_{k} b_{k}}{V_{\max }}
$$

where $V$ is the variety score; $m$ is the number of different functions to im-

prove; $f_{j}$ is the weight assigned to a certain function indicating its importance; $S_{k}$ is the weight for level $k$, Shah et al. (2003) propose 10, 6, 3, and 1 
as weights for respectively the physical principle, the working principle, the embodiment and the detail level; $b_{k}$ is the number of nodes at level $k ; V_{\max }$ is the maximum variety score obtainable. In contrast to Equation (1), Shah et al. (2003) explicitly state that, if there is only one branch at a given level, that level shows no variety and the score for that level should be zero. Furthermore, according to Shah et al., $V_{\max }$ is the variety score for the case that all ideas use different physical principles, and equals the number of concepts multiplied by the weight for the physical principle level $S_{1}$. Since $S_{1}$ equals the applied normalization factor 10, Equation (1) can be simplified as

$$
V=\sum_{j=1}^{m} f_{j} \sum_{k=1}^{4} \frac{S_{k} b_{k}}{n}
$$

where $n$ is the number of concepts. Applying Equation (2) to the genealogy tree in Figure 3 results in a variety score of $(10 * 2 / 6)+(6 * 6 / 6)=9.33$. Because higher abstraction levels are given higher weights, differentiation at higher abstraction levels leads to a higher variety score compared to the same differentiation at lower abstraction levels.

\subsection{Refinements}

In later research, Nelson et al. (2009), and Srinivasan \& Chakrabarti (2010) propose several refinements to the variety metric detailed in the sections below.

\subsubsection{Weights}

As detailed by Section 2.1, Shah et al. (2003) propose 10, 6, 3, and 1 as weights for respectively the physical principle, the working principle, the embodiment and the detail level. However, in an earlier publication, Shah 
et al. (2000) propose a slightly different set of weights: 10, 7, 4, and 1 . While this illustrates the arbitrary nature of the exact value of the weights, it also exemplifies the underlying reasoning that a set of weights must ensure a higher variety score for separation at higher levels in comparison to separation at lower levels. In line with this reasoning, Nelson et al. (2009) propose 10, 5, 2, and 1 as a different set of weights to ensure that at least two ideas at a lower hierarchical level should be added to equal the variety gained from adding an idea at a higher hierarchical level. Srinivasan \& Chakrabarti (2010) propose another set of weights based on the SAPPhIRE model, but explicitly state that these are arbitrarily chosen. Since no research provides evidence for which set of weights is preferable, a possible research trajectory encompasses the optimization of the weights to obtain a variety metric correlating strongly with the interpretation of variety by experts (see Section 5).

\subsubsection{Average variety}

Nelson et al. (2009) argue against averaging the variety metric per concept. The variety as defined by Shah et al. should however not be interpreted as an average variety, or a metric for a single concept, but as a metric for the shape of the idea space tree. A low variety is indicative of a slim tree, while a high variety indicates a wider tree, especially at higher abstraction levels. This interpretation of the variety metric is clear from Equation (1). Possibly, the interpretation as an average variety has its origins in Equation (2), in which the denominator equals the number of concepts. The denominator, of course, in turn depends on the definition of $V_{\max }$ in Equation (1).

The variety as proposed by Shah et al. was also never intended as a encompassing measure of the design space explored, as can be derived from 
following quotes (Shah et al., 2003): "This measure (variety) is necessary to counterbalance the quantity measure" and "Exploring the design space implies the number and variety of design alternatives discovered". Not averaging the variety, as proposed by Nelson et al. (2009), hence implies a redefinition of the original variety metric.

Nelson et al. (2009) further argue that a variety value can only be calculated for a set of multiple design ideas, unlike novelty, which can be calculated for a single design. From this, Nelson et al. derive that, although an average novelty score for a set of designs is a relevant metric for a set of designs, an average variety score per design is not, as the variety score only applies to the set itself. Although appealing, this deductive reasoning is flawed. The following example illustrates this by applying the reasoning proposed by $\mathrm{Nel}-$ son et al. (2009) to the unbiased estimator of the variance for a population defined as (Ross, 2004)

$$
s^{2}=\frac{1}{n-1} \sum_{i=1}^{n}\left(x_{i}-\bar{x}\right)^{2}
$$

where $s^{2}$ is the estimate of variance, $x_{1}, x_{2}, \ldots, x_{n}$ is a sample of $n$ values taken from the population, and $\bar{x}$ is the mean value of the $n$ values. Like variety, the unbiased estimator of variance is not defined for a sample containing only one value. Following the deductive reasoning proposed by Nelson et al. (2009), and since the estimator of variance is calculated by averaging as illustrated by Equation (3), it should not be used as it only applies to the set itself. However, the unbiased estimator of variance is not interpreted as an average, but as a metric on a sample set of values, just as the variety is to be interpreted as a metric on the generated design space. The fact that the 


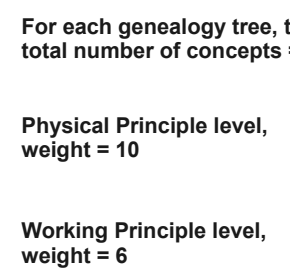

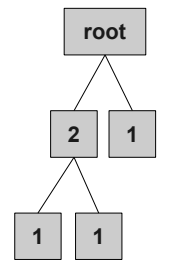

(a)

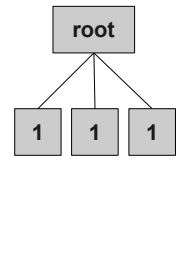

(b)

Figure 4: Examples of concept set genealogies for variety refinement discussion.

variety calculation features a division by the number of ideas hence does not preclude interpreting the variety as a metric for a design set.

\subsubsection{Single metric}

Nelson et al. (2009) propose the use of a unified metric to allow easy comparison of different ideation techniques, arguing that the use of multiple metrics makes comparison between design sets difficult. Other research applies multiple metrics (Shah et al., 2003; Linsey, 2007; Srinivasan \& Chakrabarti, 2010; Wilson et al. 2010). Based on the analysis of the effectiveness of the ideation tool PAnDA (Verhaegen et al., 2011), Peeters et al. (2010) illustrate the additional benefits of fine-grained metrics, such as a separate variety and novelty score for each abstraction level. Section 3.1 proposes a refinement to the variety metric allowing such fine-grained analysis.

\subsubsection{Double-counting ideas}

Nelson et al. (2009) illustrate flaws in formulation of the variety metric based on Figure 4. Calculating the variety with Equation (2) reveals 10.67 and 10 for respectively concept set (a) and (b). Nelson et al. argue that, since concept set (b) demonstrates the maximum variety, Equation (2) should result in a variety lower than 10 for concept set (a). Nelson et al. attribute 
this to the double-counting of ideas in Equation (2), and propose to count the number of differentiations at each node instead of the number of ideas at each level, which effectively resolves the double-counting of ideas. This modifies the variety metric to

$$
V=\sum_{j=1}^{m} f_{j}\left(S_{1}\left(b_{1}-1\right)+\sum_{k=2}^{4} \frac{S_{k} \sum_{l=1}^{b_{k-1}} d_{l}}{n-1}\right)
$$

where $d_{l}$ is the number of differentiations at node $l$, which is also equal to the number of branches emanating from node $l$ minus 1 . The division by $n-1$, equaling the maximum number of differentiations, normalizes the metric from 0 to 10 . The double-counting of ideas in a metric, however, in itself does not constitute a problem. The variety of concept set (a) exceeding 10, can also be interpreted as a consequence of an inconsistency between the numerator and denominator in Equation (2). The numerator counts the number of ideas at each level, while the denominator counts the number of concepts. Nelson et al. (2009) have adapted the numerator in Equation (2) to not count the number of ideas, but instead count the number of differentiations, which for the complete concept tree equals one less than the number of concepts.

Following Srinivasan \& Chakrabarti (2010), and consistent with the definitions of an idea and a concept given in Section 1.1, the generated ideas from all abstraction levels form an idea space, while a concept space is the collection of all generated concepts and thus includes ideas and their relationships. Since the variety metric by Nelson et al. (2009) counts the number of differentiations per node, it takes into account the relationships between ideas at different levels, which together with the ideas form the concepts. The metric proposed by Nelson et al. (2009) hence operates in concept space, and 
is therefore considered a concept space variety metric.

However, taken into account that the variety of concept set (a) exceeding 10 is caused by an inconsistency between the numerator and denominator in Equation (2), another solution is to adapt the denominator in Equation (2) to count the number of ideas in accordance with the numerator. In contrast to the concept space variety metric proposed by Nelson et al. (2009), such a variety metric does not account for the relationships between the ideas, and hence, is considered an idea space variety metric. The distinction between idea space variety and concept space variety is an important contribution made explicit in Srinivasan \& Chakrabarti (2010). Section 3.1 details a proposal for a level-based idea space variety metric based on the adaptation of the denominator in Equation (2).

\subsubsection{Variety based on SAPPhIRE}

The SAPPhIRE model was developed by Chakrabarti et al. (2005) and expresses the function, behavior and structure of systems in different abstraction levels, being $\underline{\text { State, }}$ Action, $\underline{\text { Part, }}$ Phenomena, Input, ogan and Effect (SAPPhIRE). Figure 5 illustrates how the SAPPhIRE model links these constructs through the following causal relationships: parts $(\mathrm{P})$ create organs (R), which together with various inputs (I) activate a physical effect (E), which in turn creates a physical phenomenon $(\mathrm{Ph})$ and changes the state (S) of the system. These state changes can be interpreted as actions (A), as new inputs (I), or as changes that create or activate parts (P) Chakrabarti et al., 2005).

Based on the SAPPhIRE model, Srinivasan \& Chakrabarti (2010) propose two variety scores, the idea space variety on the one hand, and the concept 


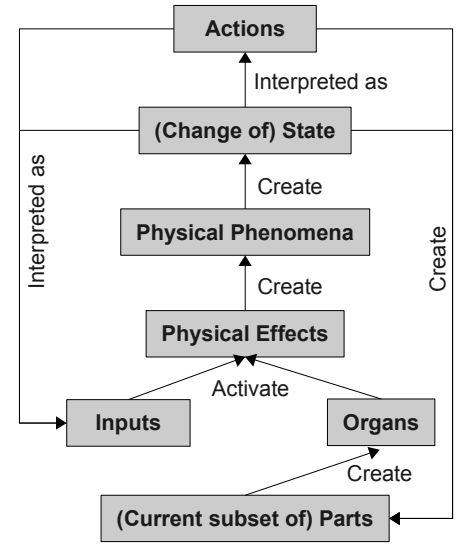

Figure 5: The SAPPhIRE model. (Chakrabarti et al. 2005)

space variety on the other. The idea space variety is calculated as

$$
V(I S)=\sum_{j=a}^{p} w_{j}\left(n_{j}-1\right)
$$

where $V(I S)$ is the idea space variety score; $j$ is summed over all abstraction levels of the SAPPhIRE model. The number of ideas at a level $j$ is $n_{j}$; and $w_{j}$ is the weight of the abstraction level $j . w_{j}$ is arbitrarily set to $7,6,5$, $4,3,2$, and 1 , for respectively the action, state change, input, phenomenon, effect, organ and part abstraction levels. If the number of ideas at a certain abstraction level $j$ is 1 , then the level's idea space variety equals zero. If the number of ideas at a certain level is 0 , then $\left(n_{j}-1\right)$ and the level's variety will become negative. Srinivasan \& Chakrabarti (2010) justify this as a penalty for skipping an abstraction level. It can be argued that such a penalty should be included in a specificity or thoroughness metric. The $\left(n_{j}-1\right)$ in Equation (5) is different from the number of differentiations in Equation (4), since Nelson et al. propose the number of differentiations per node, while Srinivasan and Chakrabarti group all ideas per abstraction level 
when calculating the idea space variety.

Srinivasan \& Chakrabarti (2010) define the concept space variety as the average of the variety of all concepts. The variety of a concept is calculated using the following steps:

(a) The first generated concept has a variety score of zero;

(b) The second generated concept is compared to the first. The idea at the highest abstraction level that discriminates the second concept from the first is identified. The weight of this abstraction level determines the variety of the second concept.

(c) In general, the $n$th generated concept is compared to all previous concepts, identifying the idea at the highest abstraction level which differentiates this concept from the $n-1$ previous ones. The weight of this abstraction level is the variety score of the $n$th concept.

The variety of a concept is clearly dependent on the order in which the concepts are generated, e.g. the first concept always receives a variety score of zero.

In contrast to the idea space genealogy trees as proposed by Shah, the $\mathrm{SAPPhIRE}$ model allows for non-tree-like idea space structures. Figure 6a depicts a section of such a non-tree-like idea space structure from Srinivasan \& Chakrabarti (2009). In this example, a lower level idea node is connected to two different higher level idea nodes, thus forming a closed path or cycle that conflicts with the characteristics of a tree. However, the above proposed concept space variety is only deterministic for tree structured idea spaces, and care should be taken when interpreting the results for non-tree-like idea spaces. A simple example without tree structure illustrates the dependency 


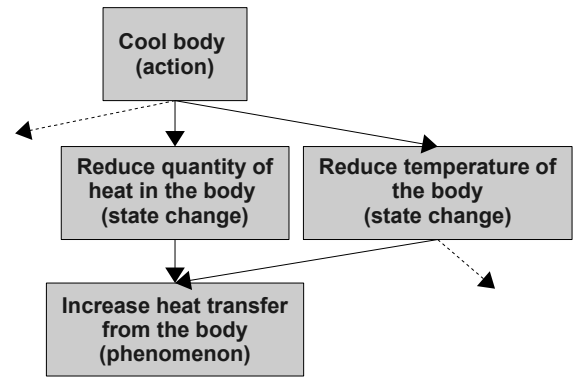

(a) Example with SAPPhIRE abstraction levels Srinivasan \& Chakrabarti, 2009)

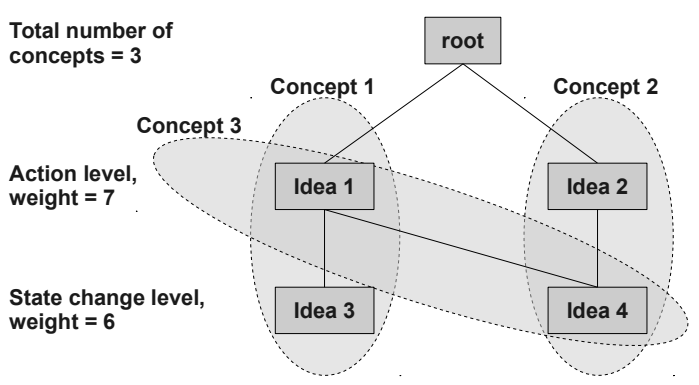

(b) Example with more generally applied abstraction levels as proposed by Shah et al. (2003)

Figure 6: Illustrations of non-tree-like idea space structures.

of the concept space variety on the generation order of the concepts. Given two abstraction levels, action and state change, with weights respectively 7 and 6, and three concepts. Each concept consists of two ideas as illustrated by the non-tree-like idea space in Figure 6b. If Concept 1 is generated first, it receives a concept variety of zero. Secondly generating Concept 2 followed by Concept 3, gives a concept variety of 7 and zero respectively. Secondly generating Concept 3 followed by Concept 2, results in a concept variety of 6 and 7 respectively. Hence, depending on the generation order, the concept space variety equals $(0+7+0) / 3=2.33$ or $(0+6+7) / 3=4.33$. It can be argued that under the assumption of a tree structured idea space, the concept space variety proposed by Srinivasan \& Chakrabarti (2010) is deterministic, and similar to the variety in Equation (4) as proposed by Nelson et al. (2009). 


\section{Proposed refinements}

\subsection{Refinement for the maximum variety and a variety measure for each level}

Section 2 illustrated a solution by Nelson et al. (2009), eliminating the double-counting of ideas and resulting in a properly normalized concept space variety metric. However, as already stated, another solution entails adapting the denominator in Equation (2) to count the number of ideas, i.e. a redefinition of $V_{\max }$ in Equation (1). Such a metric is considered an idea space variety metric, since it only takes into account the abstraction levels at which ideas occur, but does not operate on the relationships between ideas.

As stated in Section 1.2.2, the calculation of the variety requires a genealogy tree. However, the procedure to build a genealogy tree for an idea space variety metric is slightly different from the procedure to build a genealogy tree for a concept space variety metric as described hereafter. Although only defined by examples in Shah et al. (2003) and Nelson et al. (2009), it can be assumed that the genealogy trees construction is governed by the following

rule: A node of the genealogy tree has child nodes only if multiple concepts are related to this node and this node is not part of the detailed design abstraction level. Indeed, adding a child node to an existing node with only one concept would not contribute to the overall concept space variety score as the number of differentiations is zero for that node. This reasoning is supported by the counting of differentiations instead of nodes, see the double-counting refinement by Nelson et al. (2009) in Section 2.2.4.

However, for an idea space variety metric, also nodes with only one concept or idea are accounted for. Hence, the genealogy tree is extended to the 


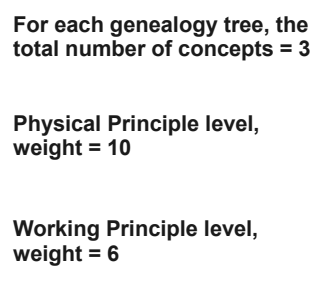

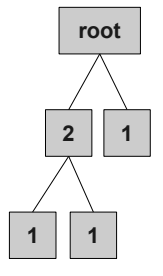

(a)

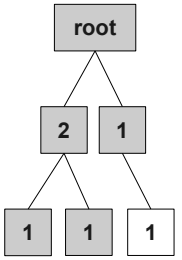

(b)

Figure 7: Genealogy trees for a concept space (a) and an idea space (b) variety metric.

lowest abstraction level. If a concept, which is unique by definition (see quantity metric in Section 1.2.1], is not detailed to the lowest level of abstraction, additional nodes representing implicit ideas are added to the genealogy tree. The addition of an implicit idea is exemplified by Figure 7 in which the node with white background in the genealogy set (b) represents the addition of an implicit idea to ensure correct idea space variety calculation. As already explained above, the concept space variety scores for genealogy sets (a) and (b) are equal if calculated based on the number of differentiations, see the double-counting refinement by Nelson et al. (2009) in Section 2.2.4. The construction of a genealogy tree with implicit nodes, furthermore, ensures that the number of nodes on an abstraction level is monotonically non-decreasing from higher to lower abstraction levels. This concurs with the assumption of a tree-like idea space, i.e. even if two concepts are only detailed to the physical principle abstraction level, it is assumed that the concepts cannot have similar ideas on lower abstraction levels. It can be argued that adding additional implicit ideas at lower abstraction levels can lead to the false impression that the concepts are detailed to this level in the concept generation phase. However, this aspect of the generated concepts is measured by the specificity or thoroughness metric (see Section 1.2.4), and it should therefore 
not be incorporated in the variety metric.

Based on a genealogy tree as illustrated by Figure 7, i.e. if needed extended with implicit ideas, it is proposed to calculate the variety for each level. This entails redefining $V_{\max }$ in Equation (1) as $V_{k_{\max }}$ being the maximum obtainable variety on level $k$. Since the genealogy tree is extended with implicit ideas if needed, $V_{k_{\max }}$ can be defined equal to the number of concepts multiplied by the weight of that level, hence $V_{k_{\max }}$ equals $n * S_{k}$. This modifies Equation (1) to

$$
V_{k}=10 \sum_{j=1}^{m} f_{j} \frac{S_{k} b_{k}}{V_{k_{\max }}}=10 \sum_{j=1}^{m} f_{j} \frac{b_{k}}{n}
$$

where $V_{k_{\max }}$ is the maximum variety for level $k, b_{k}$ equals the number of nodes on level $k$, and $n$ is the number of generated concepts. Since, as detailed above, the number of nodes is monotonically non-decreasing from higher to lower abstraction levels, $b_{k}$ and therefore $V_{k}$ in Equation (6) are monotonically non-decreasing from higher to lower abstraction levels, i.e. the variety at a given abstraction level cannot be lower than the variety of the above level.

Applying Equation (6) to the extended genealogy tree (b) in Figure 7 results in a physical level variety score of $10 *(2 / 3)=6.67$ and $10 *(3 / 3)=$ 10 for the working principle level. Equation (6) also illustrates that the maximum variety for a level is only achieved when no ideas are grouped on that level, nor on any of the levels below. This is typically the case for the lowest level of abstraction, and only if every node on that level belongs to a single concept.

Metrics at each abstraction level clearly illustrate the exact level at which 
variety is lost through the grouping of ideas, while this information is lost in a combined metric. Based on the comparison between the ideation effectiveness of brainstorming and the systematic ideation tool PAnDA (Verhaegen et al., 2011), Peeters et al. (2010) state the necessity of level-based values for the novelty metric. In contrast to the overall novelty value, which failed to discern between both ideation methods, the analysis of level-based novelty values indicated that the PAnDA tool positively affected the novelty value on the working principle level, while having little impact on the novelty value at the physical principle level. The physical level novelty value was furthermore found to be strongly correlated with the background of the participants. Without level-based metrics, the PAnDA tool would not have been identified as enhancing the novelty value. In a similar study, Peeters (2010) also report the necessity for level-based variety metrics.

\subsection{Accounting for the degree of uniformness of the distribution of ideas}

The variety defined by Shah et al. (2000), and the variety proposed by Nelson et al. (2009), both consider the genealogy set (a) and (b) in Figure 8 to have the same variety. Applying Equation (2) from Shah et al. results in a variety score of $((10 * 2)+(6 * 10)) / 10=8$ for both (a) and (b). Applying Equation (4) from Nelson et al. results in $(10 *(2-1)+6 *((5-1)+(5-1))) / 9=$ 6.4 for $(\mathrm{a})$, and $(10 *(2-1)+6 *((1-1)+(9-1))) / 9=6.4$ for $(\mathrm{b})$. The refinement from Equation (6) proposed in Section 3.1 results in a variety score of $10 *(2 / 10)=2$ for the physical principle level and $10 *(10 / 10)=10$ for the working principle level, again for both (a) and (b).

However, set (a) should have a higher variety than set (b), since the latter focuses more on a specific part of the idea space, and is thus less broad. 


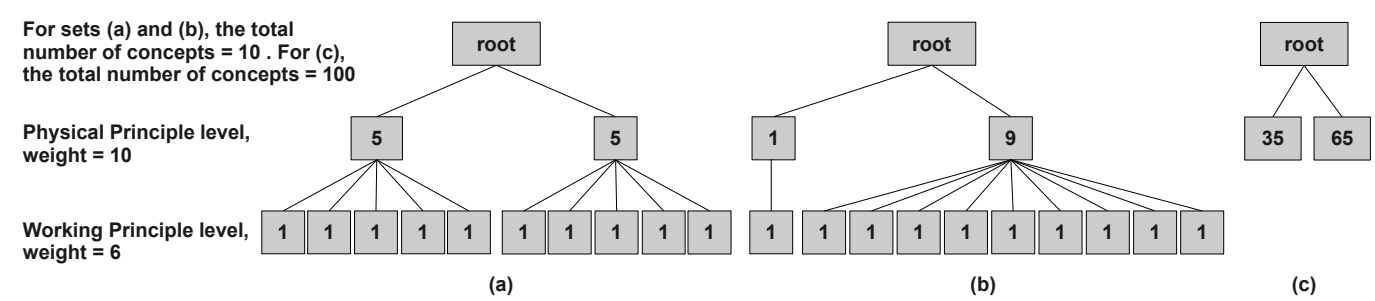

Figure 8: Example sets with different degrees of uniformness of the distribution.

This statement is supported by the following reasoning. Given a genealogy structure with only two nodes both at the physical level as illustrated by set (c) in Figure 8, Figure 9 depicts the values for the variety metric as proposed by Shah et al. (2003) for different distributions of 100 concepts over these two nodes, e.g. 35/65 on the abscissa denotes a distribution with 35 concepts in one node and the remaining 65 concepts in the other physical level node. As illustrated by Figure 9 , the variety as proposed by Shah et al. (2003) remains constant when concepts are rearranged in the idea genealogy tree, i.e. a concept is recoded from one branch to another. This leads to a constant variety score of $10 *(2 / 100)=0.2$ for all distributions except for the $0 / 100$ distribution, for which the variety score is 0.1 . The latter distribution changes the genealogy structure, i.e. the removal of one branch, triggering a sudden change from the constant value to 0.1 . In fact, the 0.1 value for the 0/100 distribution is obtained from Equation (1), although Shah et al. (2003) propose to define it to be zero. However, it is easily verified that both values result in a non-gradually changing variety score. Although the above reasoning is exemplified by the variety metric proposed by Shah et al. (2003), it applies to the variety metric proposed by Nelson et al. (2009) to the same extent. 


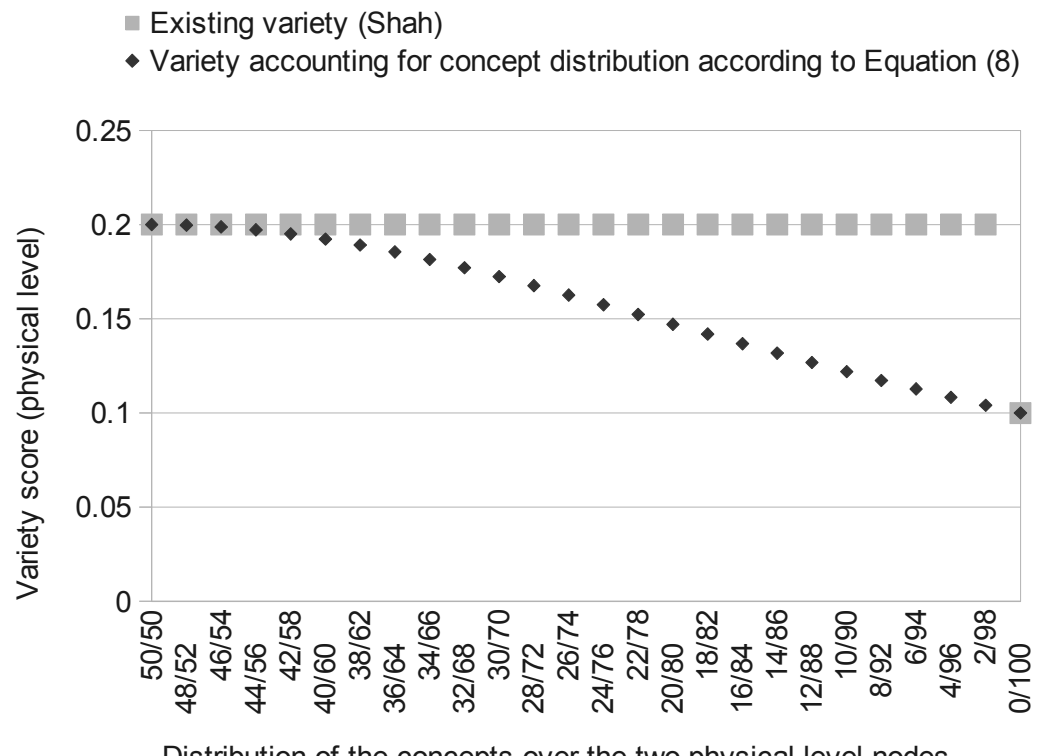

Figure 9: Illustration of the gradual behavior of the proposed refined variety metric.

In respect to the goal of the variety metric as an ideation effectiveness metric, a variety metric which changes more gradually, and is less sensitive to small changes, would be preferable. For example, an ideation tool generating an idea distribution 1/99 over the physical level nodes, and an ideation tool generating a 0/100 distribution should be considered almost identical as almost all generated concepts are alike. In fact, it can be argued that, given the uncontrollable and spontaneous nature of idea generation, it is unlikely that designers will generate concepts with a 0/100 distribution, even if the ideation method is aimed for such a distribution. Hence, a variety metric that is highly sensitive to structural genealogy changes and that does not account for the distribution of ideas can be expected to overestimate the actual variety of the idea set. It is therefore concluded that a refined variety metric gradually accounting for the distribution of concepts over nodes is preferable 
over the existing variety metrics which are only sensitive to changes in the genealogy structure.

From Equation (6), it is derived that the above described non-gradual behavior of the variety metric is caused by the factor $b_{k}$. It is proposed to replace $b_{k}$ with a factor reflecting the degree of uniformness of the distribution, gradually ranging from one to $b_{k}$ for, respectively, the case in which all ideas grouped into one branch, and the case of a uniform distribution over $b_{k}$ branches. Different measures for the degree of uniformness of a distribution are known, such as the Shannon entropy (Shannon, 1948), as well as economic measures as the Theil index (Theil, 1967), Gini index (Gini, 1997), Atkinson index (Atkinson, 1970), and Herfindahl index (Herfindahl, 1950). The latter index ranges from $1 / b_{k}$ to one, with higher scores for a non-uniform distribution. The inverse of the Herfindahl index, hence, ranges from one to $b_{k}$ with higher scores for a uniform distribution, and is consistent with the above formulated requirement. The Herfindahl index is calculated as

$$
H_{k}=\sum_{i=1}^{b_{k}} p_{i}^{2}
$$

where $H_{k}$ is the Herfindahl index at level $k ; b_{k}$ is the number of nodes at level $k ; p_{i}$ is the probability of node $i$. Substituting $b_{k}$ in Equation (6) by the inverse of the Herfindahl index leads to a level-based idea space variety metric accounting for the degree of uniformness of the distribution of ideas over nodes, which can be expressed as

$$
V_{k}=10 \sum_{j=1}^{m} f_{j}\left(\frac{1}{n H_{k}}\right)=10 \sum_{j=1}^{m} f_{j}\left(\frac{1}{n \sum_{i=1}^{b_{k}} p_{i}^{2}}\right)
$$


The variety metric proposed in Equation (8) is furthermore monotonically non-decreasing from higher to lower abstraction levels, i.e. the variety at an abstraction level cannot be lower than the variety of the above level. This concurs with the assumption of a tree-like genealogy, in which concepts with different ideas on a level cannot have similar ideas on a lower abstraction level. The monotonically non-decreasing property is easily verified based on the following reasoning. A node can have one child or multiple child nodes. In case the node has one child, the probability of that node is the same as its child node, and hence does not influence the difference between the variety score of both levels. If a parent node has multiple child nodes, the sum of the squared probabilities of the child nodes is less than the squared probability of the parent node, which leads to a lower Herfindahl index and a higher variety for the lower abstraction level in Equation (8).

Applying Equation (8) to the example set (a) in Figure 8 results in a variety score of $10 *\left(1 /\left(10 *\left((5 / 10)^{2}+(5 / 10)^{2}\right)\right)\right)=2$ for the physical principle level, and $10 *\left(1 /\left(10 *\left(10 *(1 / 10)^{2}\right)\right)\right)=10$ for the working principle level. For set (b), the variety of the physical principle level is $10 *\left(1 /\left(10 *\left((1 / 10)^{2}+(9 / 10)^{2}\right)\right)\right)=1.22$, while variety of the working principle level remains 10. From this example, it is clear that the inverse of the Herfindahl index, which replaced $b_{k}$ in Equation (6) leading to Equation (8), indeed penalizes the abstraction levels in which the ideas are more concentrated in a limited number of nodes.

Figure 9 furthermore illustrates that Equation (8) ensures a gradual changing variety score between the maximum and minimum obtainable variety with $b_{k}$ nodes for, respectively, a uniform distribution over $b_{k}$ nodes and 
an abstraction level with only one branch.

The reasoning and examples in this section illustrated the need for a variety metric that accounts for the degree of uniformness of the distribution of ideas, and although the proposed refinement based on the inverse of the Herfindahl index does resolve this issue, other measures for the degree of uniformness of a distribution might correlate better with expert variety estimations on real life data and merit further research (see Section 5).

\section{Conclusions}

The analysis of the existing variety metrics reveals several shortcomings. Firstly, a single variety metric is built based on weights assigned to the different abstraction levels. A single metric, however, lacks the necessary discriminating power to analyze and compare the results of different idea generation methods. The applied weights are, furthermore, arbitrarily assigned. Based on this analysis, it is proposed to calculate a variety measure for each abstraction level.

A second shortcoming which led to an incorrect scaling in the original variety metric proposed by Shah et al. (2003), is the inconsistent application of idea space versus concept space metrics. In addition to the concept space variety metric proposed by Nelson et al. (2009), a correctly scaling idea space variety metric is proposed encompassing a variety measure for each abstraction level.

As a third shortcoming, it is shown that the existing metrics do not measure the degree of uniformness of the distribution of ideas over nodes on a given abstraction level. It is proposed to account for this using the inverse of 
the Herfindahl index as a measure of the disorder on an abstraction level. The resulting level-based variety metric has the required discriminating power, is correctly scaled and accounts for the degree of uniformness of the distribution of ideas. In contrast to the existing variety metrics, the proposed metric is sensitive to changes in distribution of ideas over nodes on a given abstraction level in the genealogy structure, and it furthermore exhibits a monotonically increasing behavior from higher to lower abstraction levels.

\section{Future Research}

Currently, research on the ideation effectiveness metrics is limited to validating existing and proposed metrics on a set of synthetic examples. This is a necessary step, but in future research these metrics are to be validated on more elaborate sets of synthetic, and real life, idea genealogy trees supplemented with expert variety estimations. An online software tool, called

mIDGenes (Verhaegen, 2011), is being developed to allow researchers to define such synthetic and real life examples of idea genealogy trees. The tool also enables researchers to rapidly define new variety metrics, and to test these, along with the existing variety metrics, on different genealogy trees. Addition of expert opinions will allow for the optimization of the abstraction level weights to ensure the alignment of the variety metric to expert opinions.

As another research direction, and in addition to the proposed idea space metric, a level-based concept space metric accounting for the degree of uniformness of the distribution of ideas over nodes should be developed. In conjunction, different measures for the degree of uniformness of the distribution of ideas can be examined for a concept space metric, such as the Shannon in- 
formation entropy, or inequality measures for income distributions, e.g. Theil index, Gini index, or Atkinson index. It should be investigated which measures exhibit which properties, such as gradually changing, low sensitivity to small changes in genealogy structure, and monotonically increasing.

As a third research direction, it can be investigated whether real life concepts are composed of ideas from all abstraction levels, or whether abstraction levels are frequently discarded, and how this influences idea space and concept space variety metrics.

As a last research direction, the repeatability of the coding of the concepts in a genealogy tree, e.g. by different researchers, should be investigated together with robustness of the applied idea effectiveness metrics to different genealogy encodings of the generated concept set.

Altshuller, G. (1999). Innovation Algorithm: TRIZ, systematic innovation and technical creativity. (1st ed.). Technical Innovation Ctr.

Atkinson, A. (1970). On the measurement of inequality. Journal of Economic Theory, 2, 244-263.

Barton, J. A., Love, D. M., \& Taylor, G. D. (2001). Design determines $70 \%$ of cost? a review of implications for design evaluation. Journal of Engineering Design, 12, 47-58.

Bouchard, T. J. J., \& Hare, M. (1970). Size, performance, and potential in brainstorming groups. Journal of Applied Psychology, 54, 51-55.

Chakrabarti, A., Sarkar, P., Leelavathamma, B., \& Nataraju, B. (2005). A 
functional representation for aiding biomimetic and artificial inspiration of new ideas. AI EDAM, 19, 113-132.

Connolly, T., Jessup, L. M., \& Valacich, J. S. (1990). Effects of anonymity and evaluative tone on idea generation in computer-mediated groups. Management Science, 36, 689-703.

Dean, D. L., Hender, J. M., Rodgers, T. L., \& Santanen, E. L. (2006). Identifying quality, novel, and creative ideas: Constructs and scales for idea evaluation. Journal of the Association for Information Systems, 7, 646-698.

Diehl, M., \& Stroebe, W. (1987). Productivity loss in brainstorming groups - towards the solution of a riddle. Journal of personality and social psychology, 53, 497-509.

Dowlatshahi, S. (1992). Product design in a concurrent engineering environment: an optimization approach. International Journal of Production Research, 30, 1803.

Gini, C. (1997). Concentration and dependency ratios (1909) (in italian). English translation in Rivista di Politica Economica, 87, 769-789.

Girotra, K., Terwiesch, C., \& Ulrich, K. T. (2010). Idea generation and the quality of the best idea. Management Science, 56, 591-605.

Herfindahl, O. (1950). Concentration in the steel industry. Ph.D. thesis Columbia University. 
Hirtz, J., Stone, R. B., McAdams, D. A., Szykman, S., \& Wood, K. L. (2001). A functional basis for engineering design: reconciling and evolving previous efforts. Research in engineering Design, 13, 65-82.

Jansson, D. G., \& Smith, S. M. (1991). Design fixation. Design Studies, 12, $3-11$.

Linsey, J. S. (2007). Design-by-Analogy and Representation in Innovative Engineering Concept Generation. Ph.D. thesis The University of Texas Austin, Texas.

Linsey, J. S., Green, M. G., Murphy, J. T., Wood, K. L., \& Markman, A. B. (2005). Collaborating to success: An experimental study of group idea generation techniques. In ASME Design Theory and Methodology Conference (pp. 24-28).

MacCrimmon, K. R., \& Wagner, C. (1994). Stimulating ideas through creativity software. Management Science, 40, 1514-1532.

Milton, F. (2003). Innovation survey. last accessed on 01/25/2011 at http://www.wowgreatidea.com/articles/7_PWCInnovationSurvey.pdf.

Nelson, B. A., Wilson, J. O., Rosen, D., \& Yen, J. (2009). Refined metrics for measuring ideation effectiveness. Design Studies, 30, 737-743.

Osborn, A. F. (1957). Applied imagination: Principles and procedures of creative problem-solving. (3rd ed.). New York: C. Scribner's Sons.

Peeters, J. (2010). Evaluation of the effectiveness of the ideation tool 
"PAnDA" in product design. Master's thesis Katholieke Universiteit Leuven Leuven, Belgium.

Peeters, J., Verhaegen, P., Vandevenne, D., \& Duflou, J. (2010). Refined metrics for measuring novelty in ideation. In Proceedings of the IDMME Virtual Concept 2010 - Research in Interactive Design (p. 127). Bordeaux volume 3 .

Rietzschel, E. (2005). From quantity to quality: cognitive, motivational and social aspects of creative idea generation and selection. Ph.D. thesis Universiteit Utrecht.

Ross, S. M. (2004). Introduction to Probability and Statistics for Engineers and Scientists, Third Edition. (3rd ed.). Academic Press.

Shah, J. J., Kulkarni, S. V., \& Vargas-Hernandez, N. (2000). Evaluation of idea generation methods for conceptual design: Effectiveness metrics and design of experiments. Journal of Mechanical Design, 122, 377-384.

Shah, J. J., Smith, S. M., \& Vargas-Hernandez, N. (2003). Metrics for measuring ideation effectiveness. Design Studies, 24, 111-134.

Shannon, C. E. (1948). A mathematical theory of communication. The Bell System Technical Journal, 27, 379-423, 623-656.

Silverstein, D., DeCarlo, N., \& Samuel, P. (2008). The Innovator's Toolkit: 50+ Techniques for Predictable and Sustainable Organic Growth; electronic version. Hoboken, NJ: Wiley. 
Srinivasan, V., \& Chakrabarti, A. (2009). SAPPhIRE an approach to analysis and synthesis. In Proceedings of the 17th International Conference on Engineering Design (ICED'09) (pp. 417-428). Stanford, USA volume 2.

Srinivasan, V., \& Chakrabarti, A. (2010). Investigating Novelty-Outcome relationships in engineering design. Artificial Intelligence for Engineering Design, Analysis and Manufacturing, 24, 161-178.

Theil, H. (1967). Economics and information theory. Studies in mathematical and managerial economics. North-Holland Pub. Co.

Ulrich, K. T., \& Pearson, S. A. (1993). Does product design really determine $80 \%$ of manufacturing cost? Working paper Sloan School of Management Cambridge, Massachusetts.

Verhaegen, P. (2011). mIDGenes. www.mech.kuleuven.be/en/cib/s/ midgenes.

Verhaegen, P., D’hondt, J., Vandevenne, D., Dewulf, S., \& Duflou, J. R. (2011). Identifying candidates for design-by-analogy. Computers in Industry, 62, 446-459.

Weaver, J. M., Kuhr, R., Wang, D., Crawford, R. H., Wood, K. L., Jensen, D., \& Linsey, J. S. (2009). Increasing innovation in Multi-Function systems: Evaluation and experimentation of two ideation methods for design. In ASME Conference Proceedings (pp. 965-983). San Diego, California: ASME volume 2009.

Wilson, J. O., Rosen, D., Nelson, B. A., \& Yen, J. (2010). The effects of biological examples in idea generation. Design Studies, 31, 169-186. 